\title{
THE IRAM PLATEAU DE BURE INTERFEROMETER
}

\author{
Robert LUCAS \\ IRAM, Domaine Universitaire \\ 38406 Saint-Martin-d'Hères \\ FRANCE
}

ABSTRACT. We give here a brief report on the status of the IRAM Interferometer which is now in operation on Plateau de Bure.

IRAM (Institute for Radio Astronomy in the Millimeter Range) is a FrenchGerman institute, founded in 1979 by the Centre National de la Recherche Scientifique (CNRS) and the Max Planck Gesellschaft (MPG), with the contribution of the Instituto Geografico Nacional (IGN, Spain), to provide astronomers from these countries with first class facilities for millimeter wavelength astronomy. The 30-meter telescope on Pico Veleta in the Sierra Nevada has been in operation since 1985. A four element interferometer is built on the Plateau de Bure, $90 \mathrm{~km}$ south of Grenoble (France).

The site altitude is $2553 \mathrm{~m}$, in the southern French Alps. The site latitude is 44.5 degrees. Access is via a cable car operated by IRAM.

The antennas are 15 meters in diameter, with a focal ratio of 0.325 . The subreflector has a diameter of $1.5 \mathrm{~m}$. The receivers are in a cabin at the Cassegrain focus. The backup structure is made of carbon fiber tubes, and the reflecting surface consists of 176 panels (carbon fiber plates on aluminium honeycomb). The current measured accuracy of the surface is typically $60 \mu \mathrm{m}$ rms. Holographic measurements have been started, aiming to improve this figure to reach the design goal of $50 \mu \mathrm{m}$. Three of the four antennas have being built, tested, and are now regularly used for astronomy as a three-element interferometer. Tracking is good for winds speeds up to $10 \mathrm{~m} / \mathrm{s}$. The pointing accuracy is 3 " rms over the full sky. The fourth antenna is expected to be available in 1992. The antenna mounts incorporate self propelled transporters for moving the antennas along the tracks between stations. The antennas are described more completely in the contribution by Jean Delannoy, in the same volume.

The antennas may be placed on 26 stations on a T-shaped track, with arms close to the North-South and East-West directions, of lengths $160 \mathrm{~m}$ and $288 \mathrm{~m}$ respectively. The shortest possible spacing is $24 \mathrm{~m}$, to avoid collisions between 2 antennas.

There are two standard sets of configurations, named BC and CD, reasonably suited for all declinations: 
- BC gives the best available angular resolution ( $2^{\prime \prime}$ at $90 \mathrm{GHz}$ ), at the expense of observing time ( 6 observing configurations, or 18 baselines), and beam shape (elongated at low declinations).

- CD is designed for lower resolution $(\simeq 3.5$ " at $90 \mathrm{GHz})$, but gives a beam approximatively circular at all positive declinations; it uses only 5 configurations (15 baselines).

The antennas are equipped with $80-115 \mathrm{GHz}$ receivers (two SIS, one Schottky). They have DSB noise temperature between $60 \mathrm{~K}$ and $140 \mathrm{~K}$ and are tuned by remote control.

The local oscillator reference and intermediate frequencies are transported through high- $Q$ coaxial cables. The electrical lengths of the cables are monitored in real time by a round-trip phase measurement at $1.8 \mathrm{GHz}$. Phase rotators on the first and third local oscillators are used to stop the fringes; delays are digital and applied on the third local oscillators. The phase of the first local oscillator is switched by Walsh waveforms of $\pi$ and $\pi / 2$ amplitude to eliminate offsets and enable separating the upper and lower sidebands.

Two digital correlators are available:

- a continuum correlator, of total bandwith $500 \mathrm{MHz}$ per baseline (divided into ten $50 \mathrm{MHz}$ sub-bands), with 4-bit multipliers (15-level sampling, giving a higgh sensitivity of $97 \%$ ), computing simultaneously the cosine and sine components of the visibilities;

- a spectral correlator providing 128 channels per baseline. The channel spacing is adjustable between $0.156 \mathrm{MHz}$ and $1.25 \mathrm{MHz}$ (total bandwith between $20 \mathrm{MHz}$ and $2 \times 80 \mathrm{MHz}$ ). The bandwidth may be splitted in 2 subbands, positioned in the 100-600 MHz IF using 2 fixed LO frequencies taken among 150, 250, 350, 450 , and $550 \mathrm{MHz}$, used either DSB (for $80 \mathrm{MHz}$ bandwidth) or SSB (USB or LSB, for $40 \mathrm{MHz}$ and lower bandwidths). The Fourier transform and sideband separation are done in the off-line computer.

A microVAX 3400 computer and several dedicated processors control the interferometer and acquire the data. The user interface process is a variant of OBS, familiar to most users of the IRAM 30-m telescope. A second microVAX 3400 is available for first, quasi-real time calibration operations. Further calibration is done in Grenoble with a specific program (CLIC). Image construction and processing may be done either with a local package (GILDAS) or with AIPS.

First fringes were obtained on $3 \mathrm{C} 273$ and the Orion SiO maser in December 1988 , at $86 \mathrm{GHz}$ with two antennas on a $48 \mathrm{~m}$ baseline. First three-antenna operation was obtained in June 1989. The instrument has been extensively used for astronomical tests since December 1989. Regular, guest-observer operation is starting in November 1990.

Future developments include the installation of a fourth antenna, the construction of a new six-baseline correlator, of new receivers in closed cycle cryostats, with two $1.3-\mathrm{mm}$ mixers and two $3-\mathrm{mm}$ mixers, and possible baseline extension (up to $240 \mathrm{~m} \mathrm{NS}, 432 \mathrm{~m} \mathrm{EW}$ ). 


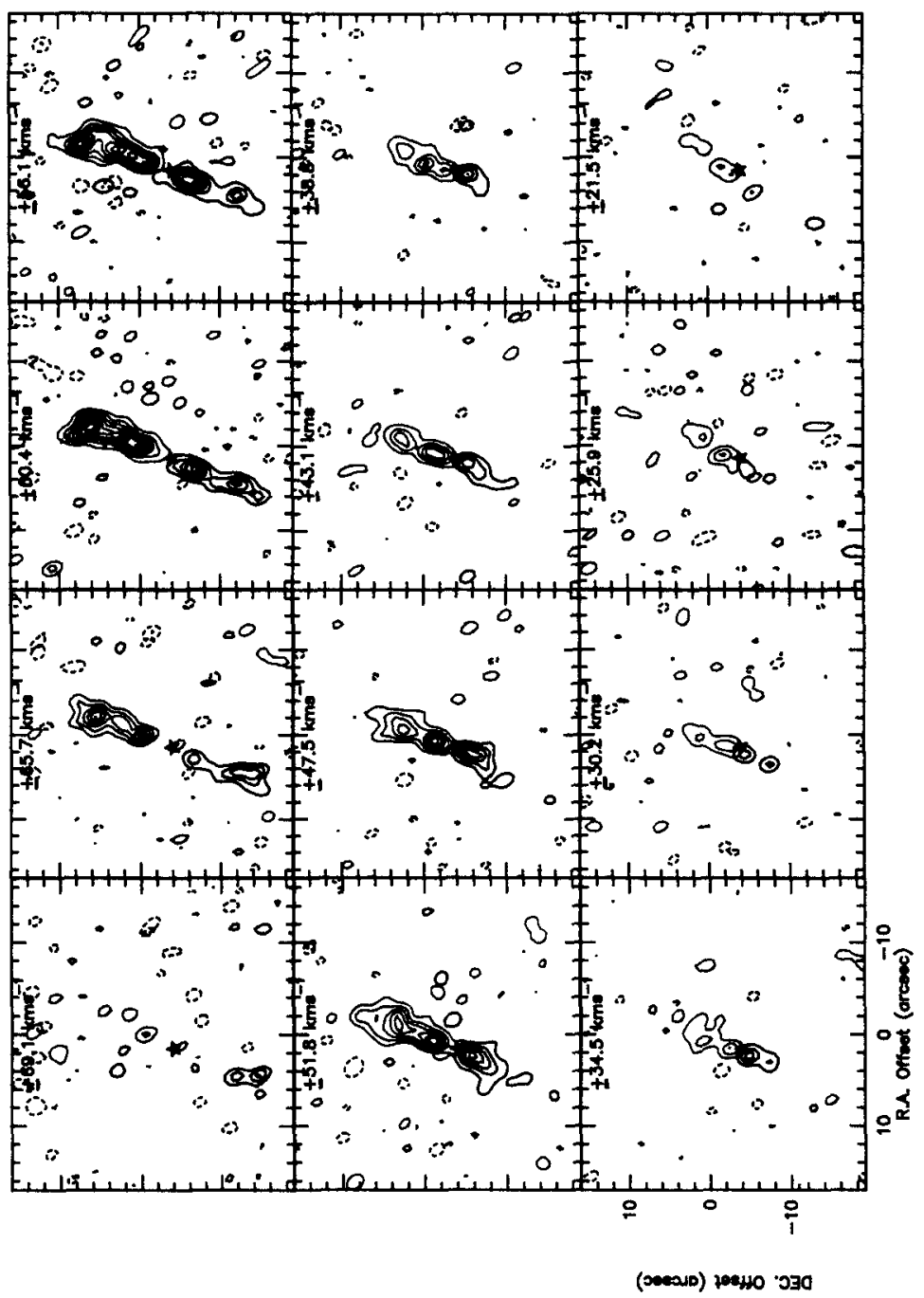

Figure 2: A 2.2" resolution systhesis map of the Lynds 1448 bipolar outflow in the $J=2-1$ rotational line of $\mathrm{SiO}$. 


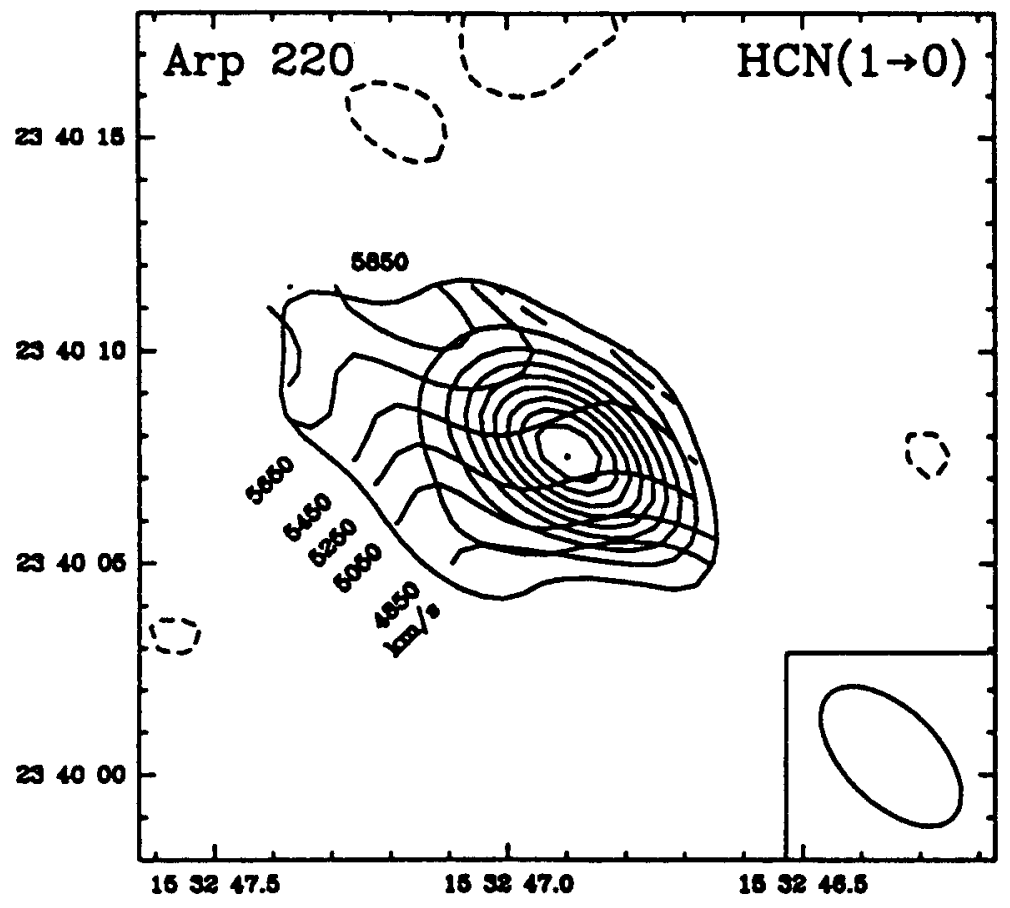

Figure 1: Integrated $\mathrm{HCN}(1-0)$ emission from the infrared luminous galaxy Arp 220 , with velocity field superimposed. 